\title{
EFFECTS OF FLOCKS DOPING ON THE DYNAMIC MECHANICAL PROPERTIES OF SHEAR THICKENING GEL
}

\author{
Yuhao Tan ${ }^{2}$, Yuling $\mathrm{Li}^{1,2, *}$, Yanxue $\mathrm{Ma}^{1,2},{ }^{*}$, Bingjun Rao ${ }^{2}$ \\ 1 Key Laboratory of Textile Science \& Technology (Donghua University), Ministry of Education, Shanghai 201620, China \\ 2 College of Textiles, Donghua University, Shanghai 201620, China \\ *Corresponding author. E-mail: Iylu@dhu.edu.cn (Y.L.L); yxma@edu.edu.cn (Y.X.M)
}

\begin{abstract}
:
Polymethyl methacrylate (PMMA) was used as the dispersed phase and mold silicone was used as the continuous phase to prepare the shear thickening gel (STG) doped with a certain amount of flocks by rotary stirring. STG doped with flocks by a rotary rheometer was tested and observed through a scanning electron microscope. Results revealed that both the rotary stirring sample preparation and the rheological test method of the rotary rheometer could lead to the regular arrangement of flocks and could not reflect shear thickening performance accurately. The flocks showed a random arrangement in STG after the mixing process, and the dynamic mechanical properties were able to be measured with a dynamic mechanical analyzer (DMA). Flocks with a fineness of 3 Denier, $1 \mathrm{~mm}$ in length, and a mass ratio of 5\% had significant effects on the dynamic mechanical properties of STG. Due to long continuous shearing time, flocks were arranged regularly when the sweeping frequency was higher than $125 \mathrm{~Hz}$, then the shear thickening performance of the doped STG was declined.
\end{abstract}

\section{Keywords:}

Shear thickening gel (STG), flocks, arrangement, dynamic mechanical properties

\section{Introduction}

As human's awareness of self-protection is gradually improving, the demand for high-quality protective products increases. Shear thickening gel (STG), which is soft and elastic, is focused as a new type of functional material [1]. STG can be used to produce civilian flexible protective materials with excellent performance, especially when it is combined with threedimensional fabrics woven by high-performance fibers [2-5]. Once impacted, the molecules lock each other quickly, tighten and harden to form a protective layer, and the three-dimensional fabric is up to fix the STG geometry and maintains the stability of the overall material. Compared with shear thickening fluids, STG is easy to encapsulate, convenient to carry, and stable in properties due to its poor liquidity. When compounded with other materials, it prevents the dispersed particles in STG from falling off the surface of the material effectively [6-8]. In addition, the preparation process of STG is relatively simple and low cost [9], which can enable large-scale industrial production. Palmer introduced shear hardening gel into high-strength polyurethane sponge and prepared a new type of human body protection material successfully [10]. Accordingly, a STG D3O has been developed, which applied non-Newtonian fluid behavior to shock-absorbing materials so that the USA ski team used D3O protective products at the 2006 Winter Olympics [11]. In recent studies, STG has exposed many problems, such as insufficient thickening effect and dependence on external environmental conditions, when it was employed. First, some STGs are prepared by dispersing the particles in a hygroscopic liquid, such as ethylene glycol, PEG200. Once the STG is exposed to the air for a long time, the shear thickening performance will be reduced because it absorbs water in the moisture $[12,13]$. Second, the STG using mold silicone as the continuous phase is less affected by the external environment, but it is found that its shear thickening performance is not significant by using a rheometer test [9]. Therefore, the thickening performance of STG needs to be further improved. Studies of the synergistic effects of short fibers compounded with polymeric matrices on the mechanical properties of composites have been reported [14]. Joel N. increased the strength and dimensional stability of rubber products by adding a small amount of pre-dispersed short fibers to the rubber compound [15]. Moreover, Yin doped chopped carbon fiber in silicone rubber to obtain a composite material with high mechanical properties [16]. Consequently, researchers have applied short fibers in flexible composites to increase toughness [17], but few research studies were carried out to investigate the effects of short fibers on the properties of STG.

Based on STG with polymethyl methacrylate (PMMA) as the dispersed phase and mold silicone as the continuous phase which has been studied by Feng [9], this study explored the effects of flocks doping on the dynamic mechanical properties of STG that doped with a certain amount of flocks. The findings of the study can be used to improve the dynamic mechanical properties of STG and provide a base for developing civil impact-resistant materials obtained by compounding STG with high-performance fabrics. 


\section{Experimental design}

\subsection{Materials}

1. Dispersed phase: Polymethyl methacrylate with a nominal particle size of $5 \mathrm{~nm}$ in spherical powder was purchased from Dongguan Zhangmutou Jinyunlai Plastic Raw Material Operation Department in Dongguan, China.

2. Continuous phase: mold silicone is 825-type translucent silicone and was purchased from Shenzhen Changdashun Trading Co., Ltd. in Shenzhen, China.

3. Flocks: flocks for electrostatic flocking were 1.5 Denier and 3 Denier polyester, with a length of $1 \mathrm{~mm}$ and were obtained from Jiashan Qida Textile Co., Ltd. in Jiaxing, China.

Flocks for electrostatic flocking are fluff fibers that are vertically attached to a fabric with an adhesive under the action of an electric field force. Neat length, uniform fineness, and certain strength are their special characteristics. At the same time, the raw materials are easily available and the cost is low. Additionally, cotton flocks, viscose flocks, and polyester flocks, and so on are introduced frequently, and the same varieties also have different lengths, fineness, and other specifications, which are commonly used fiber resources [18, 19]. In this study, a polyester flock with a length of $1 \mathrm{~mm}$ was selected, and a preliminary comparison of effects between different doping amounts and fineness of the flocks was carried out.

\subsection{STG preparation}

\subsubsection{Sample design}

Feng has found that the obtained material STG achieved the best shear thickening performance when the mass ratio of PMMA to mold silicone was $1: 1$, but the thickening effect was not significant enough [9]. Therefore, this study analyzed the shear thickening effect of STG by doping electrostatic flocking flocks with different amounts and fineness levels. The preliminary results revealed that the amount of doped flocks was greater than $10 \%$, the material would be loose and no framework could be established. Thus, the amount of doped flocks in this test did not exceed $5 \%$. Treatment combination of samples is listed in Table 1.

Table 1. Treatments combination of samples

\begin{tabular}{|c|c|c|}
\hline $\begin{array}{c}\text { Sample } \\
\text { number }\end{array}$ & $\begin{array}{c}\text { Mass ratio of PMMA/ } \\
\text { mold silicone/flocks }\end{array}$ & $\begin{array}{c}\text { Fineness of } \\
\text { flocks (Denier) }\end{array}$ \\
\hline $1 \#$ & $50 \%: 50 \%: 0$ & $/$ \\
\hline $2 \#$ & $48.75 \%: 48.75 \%: 2.5 \%$ & 1.5 \\
\hline $3 \#$ & $47.5 \%: 47.5 \%: 5 \%$ & 1.5 \\
\hline $4 \#$ & $48.75 \%: 48.75 \%: 2.5 \%$ & 3 \\
\hline $5 \#$ & $47.5 \%: 47.5 \%: 5 \%$ & 3 \\
\hline
\end{tabular}

\subsubsection{Sample preparation}

All samples were prepared in the following steps:

1. PMMA particles and flocks were placed in a vacuum drying oven (DZF, Shanghai Bangxi Instrument Technology Co., Ltd.) for $12 \mathrm{~h}$, the temperature was set to $25^{\circ} \mathrm{C}$ and the vacuum degree was controlled at $0.06 \mathrm{MPa}$ to avoid the influence of raw materials adsorbing water.

2. An appropriate amount of PMMA particles and mold silicone was weighed on an electronic balance (HBK-503H, Dongguan Zhangmutou Younet Electronic Scale Firm), and the ratio of the two was set at 1:1. Then, two types of flocks were weighed out, and the quality scores were accounted for $2.5 \%$ and $5 \%$ of total weight, respectively.

3. In the condition of heating in a $60^{\circ} \mathrm{C}$ water bath (HN-6, Shanghai Bangxi Instrument Technology Co., Ltd.), the disperse phase and continuous phase were added to the beaker successively, and a mechanical rotary stirrer (IKA RW20, Shanghai Yuxing Instrument Equipment Co., Ltd.) was used to mix and stir in $100 \mathrm{rpm}$ of the initial speed. Next, few flocks were doped successively, and the mixing speed gradually increased to $600 \mathrm{rpm}$, and the stirring time was not less than $15 \mathrm{~min}$.

4. An ultrasonic cleaner (KQ-200D, Kunshan Ultrasonic Instrument Co., Ltd.) was used to oscillate the sample for $30 \mathrm{~min}$, and the temperature was set to $60^{\circ} \mathrm{C}$ to make the distribution of phases in the doped STG more uniform and eliminate bubbles.

5. The prepared sample was placed in a vacuum drying oven for $24 \mathrm{~h}$, the temperature was set to $25^{\circ} \mathrm{C}$, and the vacuum degree was controlled at $0.06 \mathrm{MPa}$ to eliminate bubbles in depth.

\subsection{Pretest}

To explore the preparation effects of doped STG samples, the arrangement and distribution of flocks were observed under an optical microscope, and the rotary rheometer (HAAKE MARS, Thermo Fisher Scientific) was used to test the thickening performance of STG. A conical plate with a diameter of $25 \mathrm{~mm}$, an angle of $2^{\circ}$ was selected in the test, and the gap between the upper and lower plywood was $0.5 \mathrm{~mm}$. In particular, the shear rate interval was set to $0.01 \sim 100 \mathrm{~s}^{-1}$ and temperature was controlled as $25 \pm 1^{\circ} \mathrm{C}$, then the relationship between the apparent viscosity $\eta$ of the sample and the shear rate $\dot{\gamma}$ was observed.

Figure 1 illustrates the correlation between apparent viscosity and shear rate on the stirring doped STG with three different amounts of doped flocks. It can be seen that as shear rate increases, the viscosity of the STG system rises at first. When the shear rate $\dot{V}_{\text {cri }}$ is reached to a critical value, the maximum viscosity $\eta_{\max }$ appears, showing the shear thickening phenomenon at a very low shear rate in the initial stage. 


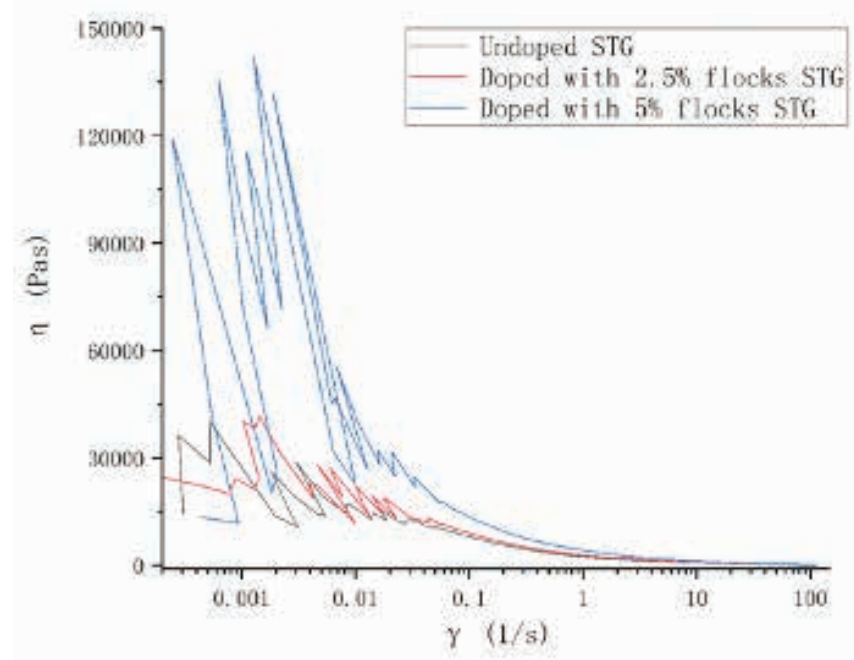

Figure 1. Rheological curves of the stirring doped STG with different amounts of doped flocks.

Nevertheless, with shear rate continues to increase, the viscosity curves rapidly decrease and finally stabilize.

The abovementioned trends reflected that the stirring doped STG might not improve the shear thickening characteristics of the system significantly, which was inconsistent with the conclusions of references [6-8]. After analyzing the causes, two improvements can be approached as follows:

1. In the preparation of the stirring doped STG, the rotation and stirring process made the spatial distribution of flocks in a circular arrangement (Figure 2), rather than a messy spatial distribution, resulting in a reduction of shear thickening effect.

2. In the rheometer test, rotation of the upper and lower splints also made the spatial distribution of flocks appear in a circular arrangement similar to rotating agitation. As rotation speed increased, the arrangement would be more regular, reducing the effect of shear thickening.

In a conclusion, it was necessary to improve the preparation method of the doped STG and choose more suitable testing instruments and methods to evaluate the doped STG system in this study.

\subsection{Improvements in preparation method}

After rotating and stirring in the fourth step of the preparation method described in Section 1.2, samples were prepared by a mechanical mixing method until the samples were colloidal, and their surfaces were no longer rough, and no obvious fine bubbles existed in the mixing doped STG [9]. It can be seen from Figure 3 that flocks are randomly arranged in the mixing doped STG. The mechanical mixing method has improved the spatial effect of the circular regular distribution to realize the heterogeneous distribution of doped flocks in the STG and provide improved conditions for a better shearing property.

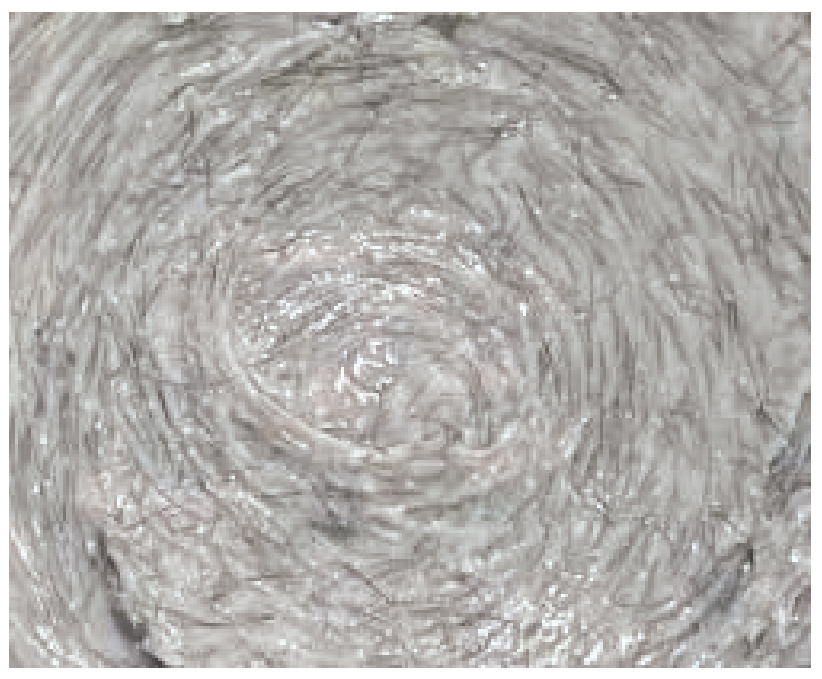

Figure 2. Image of the stirring doped STG.

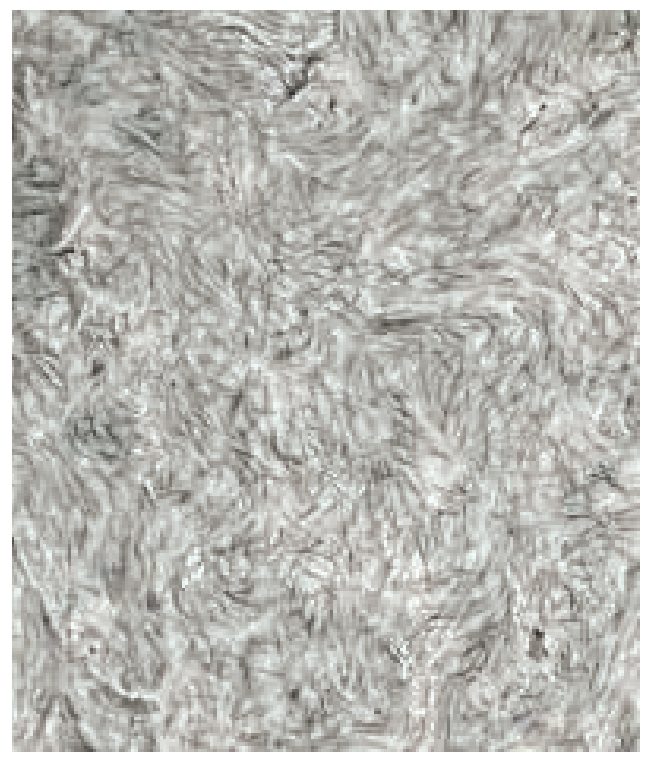

Figure 3. Image of the mixing doped STG.

\subsection{Testing methods}

\subsubsection{Morphology of STG}

Microstructures of the mixing doped STG samples were observed by scanning electron microscope (SEM; TM3000, Tianmei Technology Co., Ltd.). Before being observed, the samples were sputter-coated with gold under vacuum.

\subsubsection{Dynamic mechanical properties}

Dynamic mechanical analyzer (DMA; TA Q800, American TA Instruments) was used to test the dynamic mechanical properties, and the frequency scanning method was selected particularly. To reduce the degree of regularization of flocks in the mixing doped STG, the constant strain rate was controlled to increase shear stress to measure dynamic mechanical properties more accurate progressively. Accordingly, the indicators that characterized the dynamic mechanical properties are storage modulus G' and loss modulus G". Storage modulus is also called elastic (rigid) modulus, which refers to the amount of energy stored due to elastic (rigid) deformation when the 


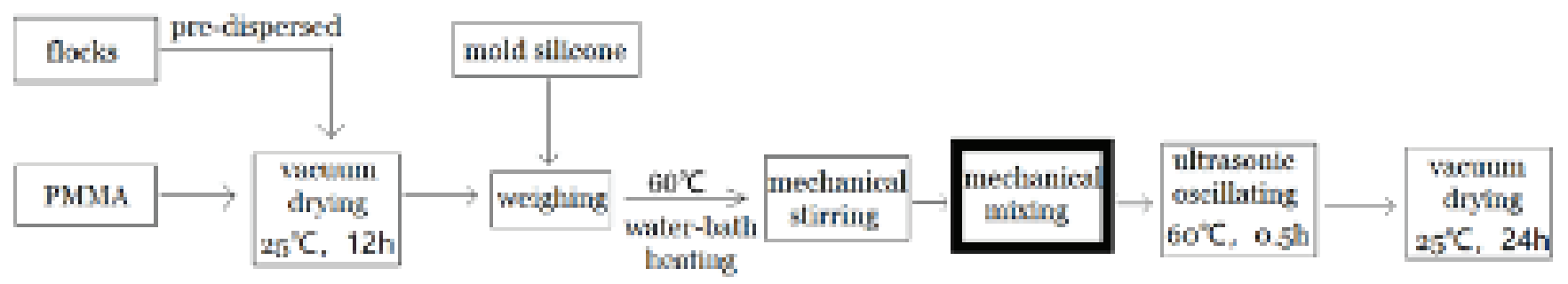

Figure 4. Flow diagram of sample preparation in the mechanical mixing process.

material undergoes deformation, reflecting the elasticity (rigidity) of the material. Loss modulus, also known as viscous modulus, refers to the amount of energy lost due to the viscous deformation of the material when it deforms, reflecting the viscosity of the material.

Because STG is flexible and unable to maintain shape, conventional fixtures could not hold the sample effectively when measured by general DMA, which made the test impossible. A new DMA test mold (Figure 5) [20] with a specification of $11 \mathrm{~mm}$ in the outer diameter of the upper cylinder and $13 \mathrm{~mm}$ in the inner diameter of the lower cylinder was designed as a fixture for testing STG and the DMA was set to tensile mode. When the temperature was $25 \pm 1{ }^{\circ} \mathrm{C}$, the strain rate was $0.5 \%$, and the frequency change interval was set to $0.1 \sim 150 \mathrm{~Hz}$, the scanning time was $80 \mathrm{~min}$, the relationship between the dynamic modulus of STG and frequency was measured.

\section{Results and discussion}

\subsection{Surface morphology of STG}

Figure 6 shows the STG surface morphology observed by SEM. It can be seen that PMMA particles are evenly distributed in mold silicone (Figure 6a). This uniform distribution ensures that the STG possessed basic mechanical properties, and few larger particles may cause the reuniting of few small particles. Compared with PMMA particles, volumes of flocks were larger and lengths of exposed ends were various. Doped flocks in the STG system were in a heterogeneous distribution and were crossed with each other. It can be clearly seen that the doped flocks had a messy spatial distribution in the mixing doped STG (Figure 6b and 6c).

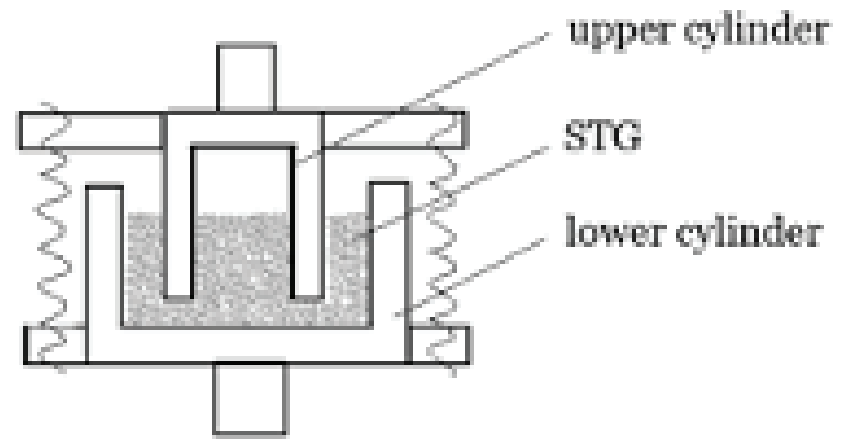

Figure 5. Sectional model diagram of new DMA test mold.

\subsection{Dynamic mechanical properties}

In the experiment, the strain rate of the mixing doped STG was $0.5 \%$ in constant, and the shear stresses were varied with different frequencies. The frequency sweep tests were conducted to analyze the influences of the amount of doped flocks on shear thickening. Figure $7(\mathrm{a})$ and (b) are storage modulus curves of the mixing doped STG with $2.5 \%$ and $5 \%$ doped flocks. It can be seen that the storage modulus of all STG samples has been greater than $300 \mathrm{KPa}$ at first, indicating that the STG samples prepared in this article have poor fluidity, which is consistent with the characteristics of the gel. Moreover, the fluidity of the doped STG and undoped STG is similar, which means the difficulty of compounding the doped STG with three-dimensional fabric is not improved significantly compared to undoped STG. Similarly, the storage modulus of all STG samples is not higher than $400 \mathrm{KPa}$ and has no significant difference in the ranges from 0.1 to $10 \mathrm{~Hz}$, indicating that doped flocks have no significant effect on the low-frequency dynamic mechanical properties of STG. This means that protective clothes made of the doped STG with three-dimensional fabrics will not interfere with the movements of the body in exercise. In addition, the storage modulus of the STG doped with flocks begins to be higher than that of the undoped STG as the frequency increases. Correspondingly, the larger the storage modulus, the greater the elasticity (stiffness) of the STG. When the frequency is higher than $20 \mathrm{~Hz}$, differences between doped and undoped STG become increasingly substantial, indicating that the influence of flocks doping on the dynamic mechanical properties of STG is at a relatively high-frequency level, which is precisely the characteristics required for flexible protective clothes [21, 22]. In the experiment, as the frequency increased, shear thickening effects were enhanced; flocks with a fineness of 3 Denier had a better performance than that of 1.5 Denier, and the doped flock amount of $5 \%$ was better than that of $2.5 \%$. However, when the shear frequency reached $125 \mathrm{~Hz}$, and the detection time was close to $1 \mathrm{~h}$, the storage modulus of the

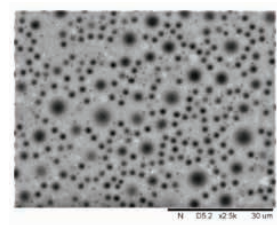

(a)

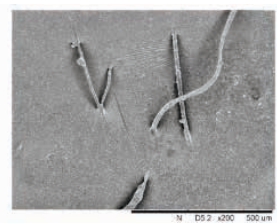

(b)

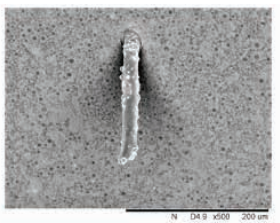

(c)
Figure 6. SEM images of STG's surface morphology. (a) morphology of the mixing doped STG without flock area; (b) and (c) morphology of the mixing doped STG containing flock regions with different magnifications. 

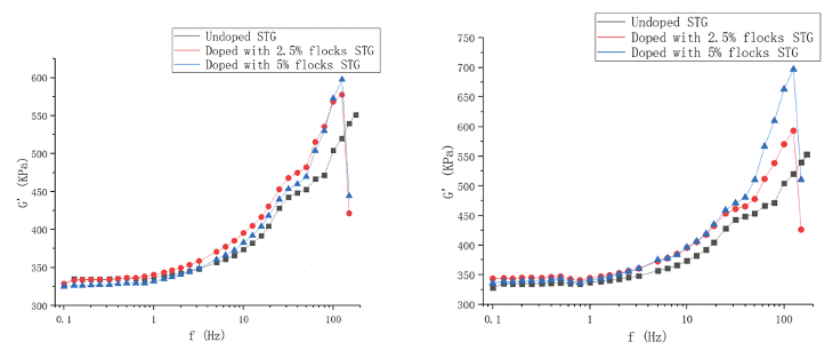

(a)

Figure 7. Storage modulus curves of the mixing doped STG with different amounts of doped flocks. (a) 1.5 Denier flocks and (b) 3 Denier flocks.

STG doped with flocks had dropped suddenly, but the undoped STG continued to increase. Therefore, this phenomenon needs to be further explored to identify whether it is the property of the STG doped with flocks or the influence of other factors.

The "particle cluster" mechanism proposed by Brady was of the opinion that the microscopic self-assembled "particle clusters" of shear thickening materials increased the viscosity of the system under the action of shear stress [23, 24]. In this study, as the shear frequency gradually increased, PMMA particles moved into mold silicone and then agglomerated, forming "particle clusters" after the STG system was doped with flocks. Due to a certain adhesiveness of the mold silicone, flocks were affected by its adhesion, and toughness of the system was enhanced by its own mechanical properties. When more mixed flocks were added to STG, the adhesive effects were more obvious. At the same time, flocks would also link or associate the clusters (Figure 8) when PMMA particles agglomerated to form "particle clusters", thereby impeding the movement of particles, the rigid energy of the STG system was enhanced, and the effect of shear thickening was further enhanced.

However, the upward trend of storage modulus curves slowed down as the shear frequency increased because the original chaotic state gradually changed after flocks were subjected to external force for a long time. Under the existing doping conditions and the duration of nearly $1 \mathrm{~h}$ and after $125 \mathrm{~Hz}$, the flocks of STG presented a higher degree of regular alignment (Figure 9) so that they could no longer play a good toughening effect. In other words, under the condition of the new DMA test

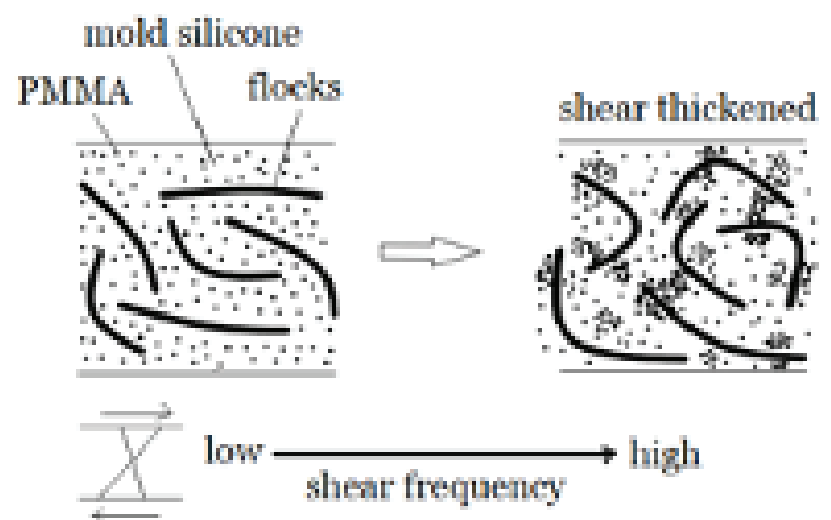

Figure 8. A diagram showing the "particle cluster" model in STG through doping with flocks. mold (Figure 5) [20], the doped STG cannot fully or completely obtain its dynamic mechanical properties through the DMA frequency sweep method. The fundamental reason should be that the duration of the test caused a regular arrangement of flocks, resulting in failure when sweeping to high frequencies.

Figure 10(a) and (b) are loss modulus curves of the mixing doped STG with $2.5 \%$ and $5 \%$ flocks. It can be seen that the loss modulus of all STG samples at the initial stage does not exceed $100 \mathrm{KPa}$, indicating that whether or not they are doped with flocks, the STG samples have a great viscosity, and their difference is not obvious. Both doped STG and undoped STG are relatively stable in morphology when they are not subjected to external forces. Similar to the storage modulus curve, the loss modulus of all STG samples is low and there is no significant difference when the frequency is ranged from 0.1 to $10 \mathrm{~Hz}$, indicating that effects of flocks doping on the performance of STG are not very obvious under the low frequency. This means that composite materials composed of doped STG and threedimensional fabrics will not affect its normal wearing and use when subjected to low-frequency external forces. Specifically, as the frequency continues to increase, the loss modulus of the doped STG increases more than that of undoped STG, indicating that the mixing doped STG has the effects of reducing vibration and energy absorption. This is because flocks were dispersed in the system and the deformation of the flocks had resistance when subjected to external forces, which in turn generated heat loss after motion friction. When more flocks doped, the energy loss would be greater. Nevertheless, the loss modulus of the doped STG begins to decrease when the frequency reaches $125 \mathrm{~Hz}$. Due to the regular arrangement of the internal flocks, the damping energy absorption function failed. In addition, the regular arrangement of flocks impeded the movement of some dispersed phase particles at high frequencies, reducing the probability of particle cluster formation. Then it resulted in the dynamic mechanical properties of the doped STG being lower than those of undoped STG.

In view of the analysis mentioned above, it was believed that $5 \%$ flocks doped in STG represented the best effect. In this level, influences of flock fineness were investigated. Figure 11 shows the storage modulus curves of the mixing doped STG with different flock finenesses. It can be seen that the storage modulus of the STG samples of each group in the lowfrequency stage does not differ significantly. Furthermore, the storage modulus of the flocks fineness of the 3 Denier group is higher than the 1.5 Denier group after reaching $20 \mathrm{~Hz}$,

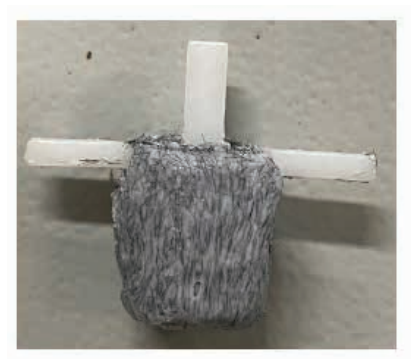

(a)

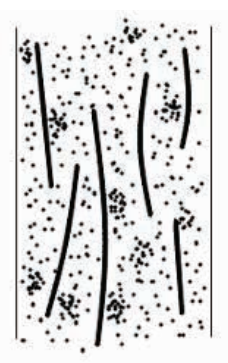

(b)
Figure 9. Two images showed a higher degree of flocks arranged in the same direction: (a) physical image and (b) model image. 


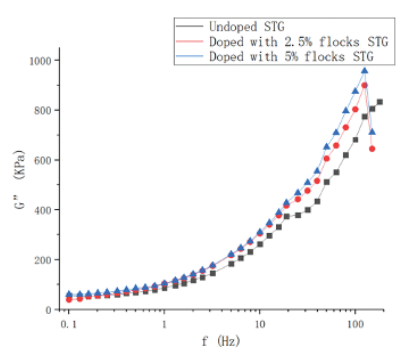

(a)

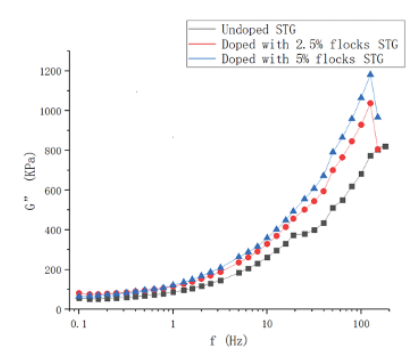

(b)
Figure 10. Loss modulus curves of the "mixing doped STG" with different doping amounts: (a) the fineness of flock is 1.5 Denier and (b) the fineness of flock is 3 Denier.

and the gap increases gradually, indicating that the larger fineness of flocks can promote the system to store energy so that the rigidity of the mixing doped STG becomes larger. This phenomenon is caused by the fineness of 3 Denier flocks with better mechanical properties. When combined with the mechanism of flocks in the mixing doped STG system, it can be considered that the fineness of $3 \mathrm{D}$ polyester flocks improved the toughness of the system better. However, when the sweeping frequency reaches $125 \mathrm{~Hz}$ and the duration is close to $1 \mathrm{~h}$, the storage modulus of the doped STG decreases suddenly, and this decrease in the fineness of 3 Denier doped STG is not as notable as that of 1.5 Denier. The reason is that the shortness of large fineness was worse, and the movement in the STG system was not as flexible as that of small fineness. Therefore, the high-frequency effect made the shortness of 1.5 Denier flocks arranged more regularly, thereby the failure of toughening effect was more significant.

\section{Conclusions}

The doped flocks can improve the storage modulus and loss modulus of the STG system, and the enhancement of the dynamic mechanical properties of the doped STG would be more significant with the larger doping amount or fineness. This discovery provides a way to improve the lack of existing STG shear thickening performance. Additionally, the optimal shear thickening effect can only be obtained when the doped flocks were arranged in a random arrangement and in various directions. Similarly, the preparation method of STG also affected its shear thickening properties notably. Therefore, existing methods for detecting rheological properties and dynamic mechanical properties all have the problem of interfering with the disordered distribution of flocks significantly in the doped STG. This makes it difficult to accurately or fully characterize the shear thickening characteristics of the doped STG. This article innovatively proposes the use of DMA to test the dynamic mechanical properties of STG doped with flocks, which has certain guiding significance for future research in this direction.

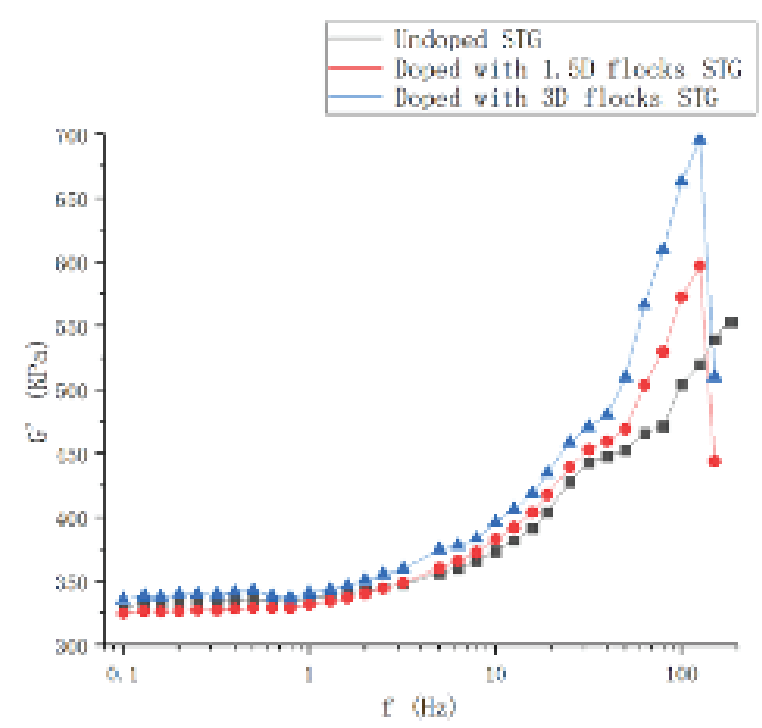

Figure 11. Storage modulus curves of the mixing doped STG with different fineness.

\section{ACKNOWLEDGEMENTS}

Funding from Fundamental Research Funds for the Central Universities (2232020G-01), The National Key Research and Development Project (2017YFB0309200) and The National Natural Science Foundation of China (No. 71704021) are gratefully acknowledged.

\section{References}

[1] Houston, J. E. (2005). A local-probe analysis of the rheology of a "solid liquid". Journal of Polymer Science Part B: Polymer Physics, 43(21), 2993-2999.

[2] Maguz, H., Clements, F., Rangari, V., Dhanak, V., Beamson, G. (2009). Enhanced stab resistance of armor composites with functionalized silica nanoparticles. Journal of Applied Physics, 105, 064307-064314.

[3] Lee, Y. S., Wetzel, E. D., Wagner, N. J. (2003). The ballistic impact characteristics of Kevlar woven fabrics impregnated with a colloidal shear thickening fluid. Journal of Materials Science, 38, 2825-2833.

[4] Afeshejani, S. H., Sabet, S. A., Atai, M. E. (2014). Energy absorption in a shear-thickening fluid. Journal of Materials Engineering and Performance, 23, 4289-4297.

[5] Soutrenon, M., Michaud, V. (2014). Impact properties of shear thickening fluid impregnated foams. Smart Materials and Structures, 23, 035022.

[6] Lin, N. C., Guy, B. M., Hermes, M., Ness, C., Sun, J., et al. (2015). Hydrodynamic and contact contributions to continuous shear thickening in colloidal suspensions. Physical Review Letters, 135, 228304-228309.

[7] Yu, K. J., Cao, H. J., Qian, K., Sha, X., Chen, Y. (2012). Shear-thickening behavior of modified silica nanoparticles in polyethylene glycol. Journal of Nanoparticle Research, 14, 747-756.

[8] Wang, S., Xuan, S. H., Wang, Y. P., Xu, C. H., Mao, Y., et al. (2016). Stretchable polyurethane sponge scaffold strengthened shear stiffening polymer and its enhanced safeguarding performance. ACS Applied Materials and interfaces, 8, 4946-4954. 
[9] Feng, R. R. (2020). Study on the puncture resistance of fabrics with integral pleated structure reinforced by shear thickening fluid. MD Thesis, Donghua University, China.

[10] Green, P., Palmer, R. (2010). USA, US0132099A12010.

[11] Guo, Z. C. (2016). DuPont and British D3O develop new multi-purpose plastic protective materials. Chemical Propellants \& Polymeric Materials, 14(04), 68.

[12] He, Q. Y., Cao, S. S., Wang, Y. P., Xuan, S., Wang, P., et al. (2017). Impact resistance of shear thickening fluid/Kevlar composite treated with shear-stiffening gel. Composites: Part A, 106, 82-90.

[13] Li, D. Y., Wang, R., Liu, X., Zhang, S., Fang, S., et al. (2020). Effect of dispersing media and temperature on inter-yarn frictional properties of Kevlar fabrics impregnated with shear thickening fluid. Composite Structures, 249, 112557112566.

[14] Natalia, M., Wladyslaw, M. R. (2013). Use of short fibers as a filler in rubber compounds. AUTEX Research Journal, 2(13), 40-43.

[15] Joel, N., Steven, M., Cristian, O. (2019). Pre-dispersed short fibers: A cost-effective way to reinforce rubber compounds. Advanced Rubber Technology, 2, 35-40.

[16] Yin, S., Ma, L., Wu, L. Z. (2011). Carbon fiber composite lattice structure filled with silicone rubber. Procedia Engineering, 10, 3191-3194.
[17] Budsaraporn, S., Samar, H., Gautier, S., Karine, M., Taweechai, A. (2020). Comparative study of pineapple leaf microfiber and aramid fiber reinforced natural rubbers using dynamic mechanical analysis, Polymer Testing, 82, 106289-106299.

[18] Chen, X. Y., Gu, Y. Z., Liang, J. Y., Bai, M., Wang, S., et al. (2020). Enhanced microwave shielding effectiveness and suppressed reflection of chopped carbon fiber felt by electrostatic flocking of carbon fiber. Composites Part A: Applied Science and Manufacturing, 139, 106099-106110.

[19] Kim, Y. K. (2011). Specialist yarn and fabric structures. Woodhead (Cambridge, UK), pp. 287-317.

[20] Li, Y. L., Rao, B. J., Tan, Y. H. (2019). A new DMA mold for testing the mechanical properties of fluids: China, CN110617944A. Oct. 25, 2019.

[21] Wetzel, E. D., Lee, Y. S., Egres, R. G. (2004). The effect of rheological parameters on the ballistic properties of shear thickening fluid (STF)-Kevlar composites. American Institute of Physics (New York, USA), pp. 288-293.

[22] Anna, W., Piotr, D. (2016). Research on biophysical properties of protective clothing, AUTEX Research Journal, 4(16), 236-240.

[23] Brady, J. F., Bossis, G. (1985). The rheology of concentrated suspensions of spheres in simple shear flow by numerical simulation. Journal of Fluid mechanics, 29, 145-155.

[24] Brady, J. F., Bossis, G. (1988). Stokesian dynamic annual review of fluid mechanics. Journal of Fluid mechanics, 201, 41-57. 